\title{
Das Bewegungssehen der Fliege: vom optischen Fluss zur visuellen Kurskontrolle
}

\author{
Alexander Borst
}

\section{Zusammenfassung}

Visuelle, auf dem optischen Fluss basierende Kurskontrolle wurde sowohl an stationären als auch an frei fliegenden Fliegen ausgiebig studiert. Für die neuronale Kontrolle dieses Verhaltens scheinen die Tangentialzellen der Lobulaplatte eine große Rolle zu spielen. Diese Zellen sind bewegungsempfindlich, haben große rezeptive Felder, und die räumliche Verteilung ihrer Vorzugsrichtungen entspricht dem optischen Fluss, wie er bei bestimmten Flugmanövern auftritt. Der präsynaptische Schaltkreis, der die lokalen Bewegungsrichtungen berechnet und die Tangentialzellen überhaupt erst empfindlich für visuelle Bewegung macht, lag jedoch lange Zeit aufgrund der geringen Größe der entsprechenden Neurone im Dunkeln. Erste Erfolge erbrachte hier die Kombination von elektrophysiologischen Ableitungen der Tangentialzellen bei Drosophila mit genetischer Blockade einzelner Kandidaten-Neurone. Mit diesem Ansatz wurden die Lamina-Neurone L1 und L2 als Eingangselemente zu zwei parallelen Bewegungsdetektionssystemen identifiziert, die selektiv auf die Bewegung von Dunkel-Hell (L1, ON-System) bzw. Hell-Dunkel (L2, Off-System) -Übergängen reagieren.

\begin{abstract}
Fly Motion Vision: From Optic Flow to Visual Course Control.

Optic-flow based navigation has been studied extensively in flies, both in tethered as well as in freely flying animals. As neural control elements, the tangential cells of the lobula plate seem to play a key role: they are sensitive to visual motion, have large receptive fields, and, with their spatial distribution of preferred directions, match the optic flow as elicited during certain types of flight maneuvers. However, the neural circuit presynaptic to the tangential cells responsible for extracting the direction of motion locally has escaped for long, due to the small size of the participating neurons. Here, recent progress was made by combining genetic silencing of candidate neurons with whole-cell patch recording from tangential cells in Drosophila. This approach led to the identification of lamina neurons $L 1$ and $L 2$ providing the input signals to two parallel motion detection circuits, specialized for brightness increments (L1, ON-pathway) and decrements (L2, OFF-pathway), respectively.
\end{abstract}

Keywords: insects; optic flow; motion vision; neural information processing; course control

\section{Einleitung}

Wenn eine Fliege herumfliegt, bewegen sich andauernd Bilder der Umgebung über ihre Retina. Die Verteilung der Bewegungsvektoren im Gesichtsfeld des Tieres nennt man optischen Fluss (Abbildung 1, links). Der optische Fluss enthält eine Fülle an Informationen über den Kurs der Fliege und wird deshalb für die visuelle Kurskontrolle genutzt. Bei der Fliege werden visuelle Signale in mehreren aufeinanderfolgenden Neuropilschichten, der Lamina, der Medulla, der Lobula und der Lobulaplatte, verarbeitet
(Abbildung 1, Mitte). Die weitaus wichtigste Verarbeitung auf diesem Weg ist die Berechnung lokaler Bewegungsvektoren durch elementare Bewegungsdetektoren. Dadurch wird der optische Fluss durch einen ,neuronalen Fluss ‘ repräsentiert. Diese Signale werden in der Lobulaplatte durch eine Gruppe großer Tangentialzellen räumlich zusammengefasst. Tangentialzellen reagieren oft spezifisch auf einen bestimmten optischen Fluss. Ihr Ausgangssignal kann daher direkt für kompensatorische Kopfbewegungen oder Kurskorrekturmanöver genutzt werden (Abbildung 1, rechts).

\section{Elementare Bewegungsdetektion}

Das Fliegengehirn widmet einen Großteil seines Neuropils der Verarbeitung der Bilder, die es von seinen Facettenaugen erhält. Dieser Teil des Gehirns, der optische Lobus, wird in mehrere Schichten unterteilt, nämlich die Lamina, die Medulla, die Lobula und die Lobulaplatte. Diese Schichten sind aus repetitiven Säulen aufgebaut und bilden die visuelle Umgebung retinotop ab. Mithilfe der Golgi-Methode wurde von Ramon y Cajal und Sanchez (1915) die Anatomie des optischen Lobus der Fliege erstmals mit zellulärer Auflösung beschrieben. Später folgten weitgehend vollständige Beschreibungen des optischen Lobus der Hausfliege Musca domestica und der Taufliege Drosophila melanogaster (Fischbach und Dittrich 1989) ebenfalls unter Verwendung der GolgiMethode. Über die verschiedenen Schichten verteilt besteht eine Säule aus ungefähr 100 Neuronen. Auf der Ebene der Lobulaplatte fasst eine Gruppe von großen Weitfeldneuronen, die sogenannten Tangentialzellen, die Ausgangssignale von Hunderten solcher Säulenelemente zusammen.

Die wichtigste Antworteigenschaft der Tangentialzellen der Lobulaplatte ist ihre Richtungsselektivität: Wenn ein Muster sich in eine Richtung bewegt (in die Vorzugsrichtung der Zelle), depolarisiert die Zelle oder entlädt eine Serie von Aktionspotenzialen. Wenn sich ein Muster in die entgegengesetzte Richtung bewegt (die Nullrichtung der Zelle), hyperpolarisiert die Zelle oder hört auf zu feuern. Im Gegensatz dazu ist das Signal der Photorezeptoren nicht richtungsselektiv, d.h. ein individueller Photorezeptor gibt die gleiche Antwort auf einen Lichtreiz unabhängig davon, in welche Richtung sich der Reiz bewegt. Irgendwie wird also eine richtungsunabhängige Antwort auf der Ebene der Photorezeptoren in eine richtungsselektive Antwort auf der Ebene der Tangentialzellen in der Lobulaplatte überführt. Diese Transformation wird durch ein bestimmtes Modell, den sogenannten „Reichardt-Detektor", phantastisch genau beschrieben.

Das Reichardt-Modell für elementare Bewegungsdetektion wurde ursprünglich anhand von Untersuchungen der optomotorischen Reaktion des Käfers Chlorophanus entwickelt, der auf einem Spangenglobus lief, während er von einem periodischen $\mathrm{Mu}$ ster umgeben war (Hassenstein und Reichardt 1956). In diesem Modell (Abbildung 2A) werden die Signale von benachbarten Photorezeptoren multipliziert, nachdem eines von ihnen durch einen zeitlichen Tiefpassfilter verzögert wurde. Dieser Vorgang 
Abb. 1: Die Eigenbewegung der Fliege verursacht einen optischen Fluss, der über die Facettenaugen auf mehrere Schichten von Neuropil vermittelt wird. Innerhalb dieser Schichten wird von einer zweidimensionalen Anordnung von Bewegungsdetektoren die Verteilung der Bewegungsvektoren (neuronaler Fluss) berechnet. Ihre Ausgangssignale treffen auf eine Gruppe großer Tangentialzellen, die jeweils spezifisch auf ein bestimmtes Flussfeld reagieren. Die Tangentialzellen kontrollieren kompensatorische Kopfbewegungen und Steuerungsmanöver der Fliege.

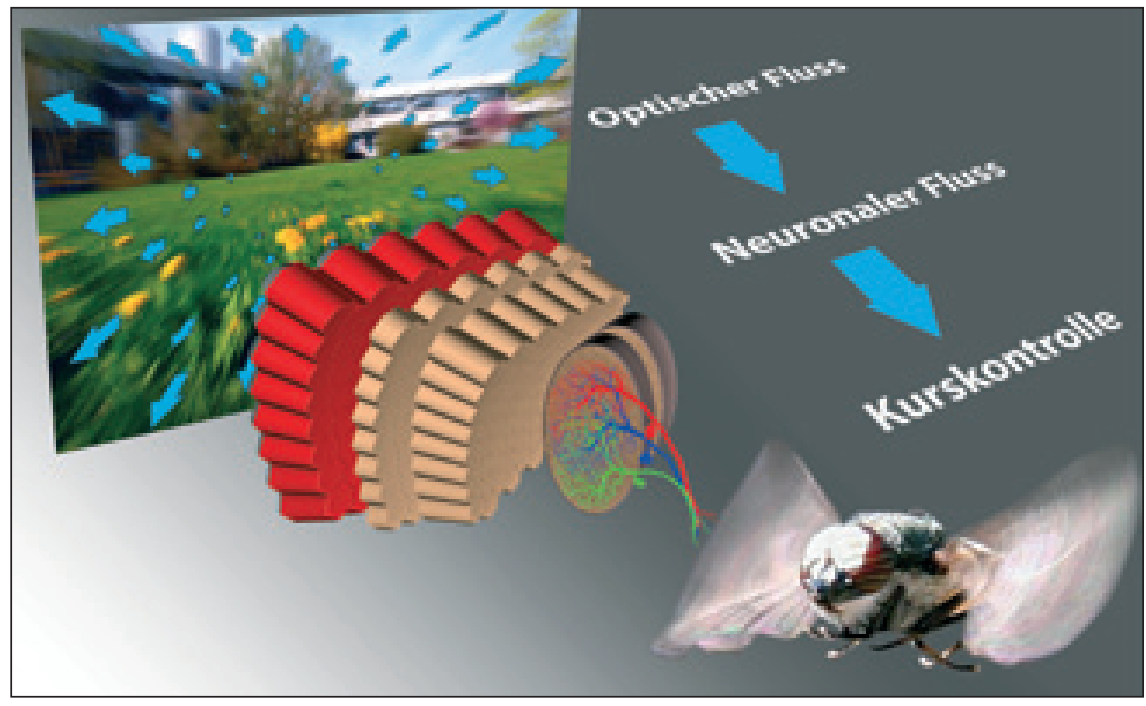

Anordnung solcher Elementardetektoren, die jeweils nur ein kleines rezeptives Feld haben, das gesamte Gesichtsfeld abdeckt. Die beobachtete optomotorische Antwort wird dann ausgelöst, indem die Ausgangssignale dieser lokalen Bewegungsdetektoren räumlich zusammengefasst werden. Dieses Modell führt $\mathrm{zu}$ einigen wenig intuitiven Vorhersagen: 1. Die Antwortstärke sollte mit zunehmendem Kontrast des Musters zunehmen. 2. Im Gegensatz zu einem Tachometer sollte die Antwort ein Geschwindigeine richtungsselektive Antwort. Die pothese ist nun, dass eine zweidimensionale

Untereinheiten durchgeführt. Die Ausgangssignale der beiden Untereinheiten werden dann voneinander subtrahiert, es entsteht

\section{Ausschreibung für den}

\section{FELIX-WANKEL-TIERSCHUTZ- FORSCHUNGSPREIS 2013}

Der Felix-Wankel-Tierschutz-Forschungspreis wird durch die Ludwig-Maximilians-Universität München in der Regel alle zwei Jahre für hervorragende, experimentelle und innovative wissenschaftliche Arbeiten verliehen, deren Ziel bzw. Ergebnis es ist, Tierversuche zu ersetzen oder einzuschränken, den Tierschutz generell zu fördern, die Gesundheit und tiergerechte Unterbringung von Versuchs-, Heim- und Nutztieren zu gewährleisten oder die Grundlagenforschung zur Verbesserung des Tierschutzes zu unterstützen.

\section{Der Preis ist mit maximal 30000 EURO dotiert.}

Eine Aufteilung des Preises auf mehrere Preisträger ist möglich. Die Verwendung des Preisgeldes ist nicht mit Auflagen verbunden. Vorschlagsberechtigt sind Wissenschaftler sowie Mitglieder zum Beispiel von wissenschaftlichen Institutionen, von Fachgesellschaften und von Behörden sowie von Wissenschaftsredaktionen. Vorgeschlagen werden können Personen und Gruppen, die in der Forschung im In- oder Ausland tätig sind. Die Arbeiten sollen neueren Ursprungs sein und eigene Forschungsergebnisse enthalten. Sie müssen im Druck vorliegen. Bereits anderweitig mit einem Tierschutzpreis ausgezeichnete Arbeiten werden in der Regel nicht berücksichtigt. Eine Eigenbewerbung ist ausgeschlossen.
Mit dem Vorschlag müssen die Arbeiten in dreifacher Ausfertigung eingereicht werden. Zusätzlich sind in elektronischer Form (PDF-Datei) auf Diskette oder CD-ROM Lebenslauf, Schriftenverzeichnis und eine maximal zweiseitige Kurzfassung in deutscher und/oder englischer Sprache vorzulegen, die den Stand des Wissens, den Forschungsansatz und die Ergebnisse darstellt. Ein Exemplar der vorgelegten Arbeiten bleibt bei den Akten des Kuratoriums.

Die Vorschläge mit den Arbeiten müssen bis 30. September 2012 bei der Geschäftsstelle für den Felix-Wankel-TierschutzForschungspreis an der Tierärztlichen Fakultät der LudwigMaximilians-Universität München vorliegen. Über die Zuerkennung des Preises entscheidet das Kuratorium des FelixWankel-Tierschutz-Forschungspreises; sie erfolgt unter Ausschluss des Rechtsweges.

Informationen zum Felix-Wankel-Tierschutz-Forschungspreis auch im Internet über http://www.felix-wankel-forschungspreis.de Weitere Auskünfte erteilt die Geschäftsstelle für den

Felix-Wankel-Tierschutz-Forschungspreis am Lehrstuhl für Tierschutz, Verhaltenskunde, Tierhygiene und Tierhaltung, Veterinärwissenschaftliches Department der Tierärztlichen Fakultät,

Ludwig-Maximilians-Universität München, Veterinärstr. 13/R, 80539 München, Tel. + 4989218078300 , Fax +49 89218078333 , Email: felix.wankel@tierhyg.vetmed.uni-muenchen.de 

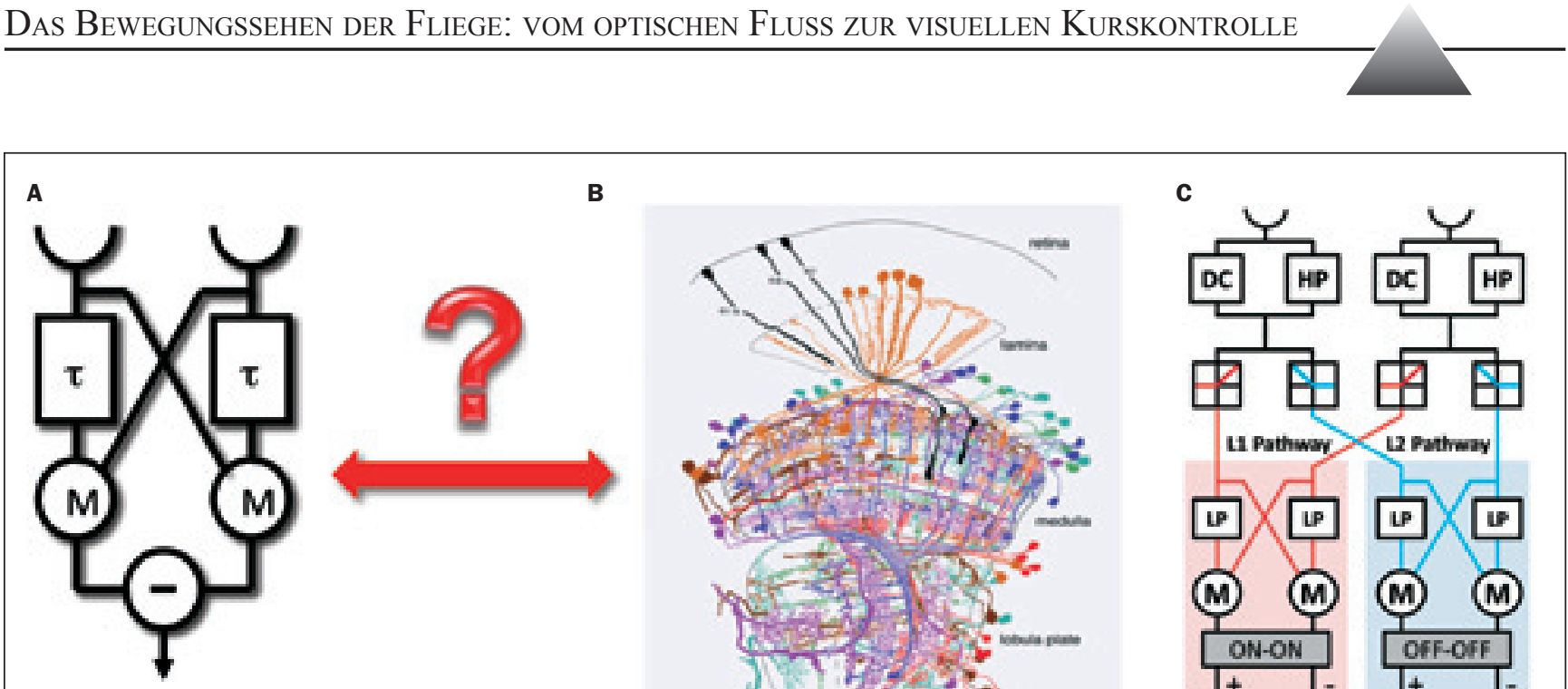

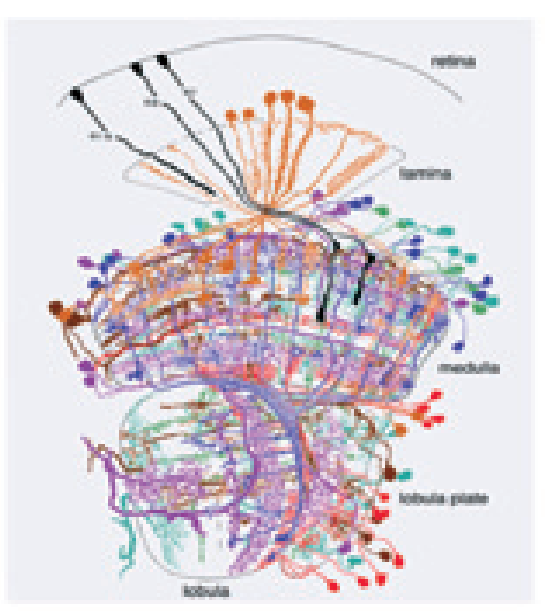

C

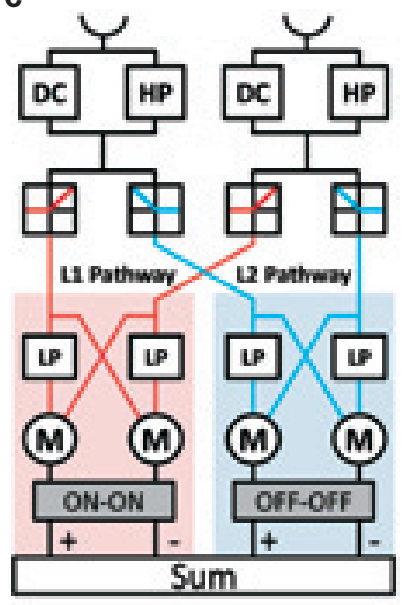

Abb. 2 A): Der Reichardt - Detektor ist ein Algorithmus, der beschreibt, wie lokale Bewegungsinformation aus nicht-richtungsselektiven Eingangssignalen extrahiert wird. Er besteht aus zwei spiegelsymmetrischen Untereinheiten, die jeweils Eingang von benachbarten Photorezeptoren erhalten. Innerhalb einer jeden Untereinheit wird das Signal eines Photorezeptors durch einen Tiefpassfilter mit einer Zeitkonstante $\tau$ verzögert und anschließend mit dem augenblicklichen Signal des benachbarten Photorezeptors multipliziert (M). Die Ausgangssignale der beiden Untereinheiten werden anschließend voneinander subtrahiert. B): Die neuronalen Säulen des optischen Lobus der Fliege (nach Fischbach und Dittrich 1989). Ein Großteil der heutigen Forschung über das Bewegungssehen der Fliege befasst sich mit der Identifikation derjenigen Neurone in den Säulen, die die Komponenten des Reichhardt - Detektors repräsentieren. Lamina, Medulla, Lobula und Lobulaplatte (lobula plate) sind Schichten des optischen Lobus. C): Unter den verschiedenen Neuronen der Lamina der Fliege repräsentieren L1 und L2 die Eingangslinien zum Bewegungssehsystem. Durch die Aufteilung des Eingangssignals in ein ON (L1)- und ein OFF-Signal (L2) entsteht eine funktionelle Spezialisierung. In diesen beiden Systemen wird Bewegung parallel verarbeitet. Die Fliege besitzt also 2 Bewegungsdetektoren pro Säule, einen, der die Bewegung von Dunkel-Hell-Kanten verarbeitet, und einen für die Bewegung von Hell-Dunkel-Kanten. DC = ,Direct Current" = Gleichspannungs-Anteil, HP = ,High-Pass" = Hochpass, LP = ,Low-Pass“ = Tiefpass, $\mathbf{M}=$ Mulitplikation

keitsoptimum haben. Jenseits der optimalen Geschwindigkeit sollte die Antwortstärke mit steigender Geschwindigkeit abnehmen. 3. Muster mit verschiedenen räumlichen Wellenlängen sollten unterschiedliche Geschwindigkeitsoptima haben, sodass das Verhältnis aus optimaler Geschwindigkeit und der räumlichen Wellenlänge, welches die Dimension einer zeitlichen Frequenz hat, konstant bleibt.

Nicht nur die optomotorische Reaktion, sondern auch die visuellen Antworten der Tangentialzellen in der Lobulaplatte der Schmeißfliege und der Taufliege (Schnell et al. 2010) zeigen diese Eigenschaften. Bei beiden Spezies liegt die optimale zeitliche Frequenz bei etwa $1 \mathrm{~Hz}$. Im Gegensatz zur optomotorischen Reaktion, die von Natur aus langsam ist, konnte man bei den Ableitungen der Tangentialzellen der Schmeißfliege die Antworttransienten der zellulären Antworten mit denen des Reichardt-Detektors vergleichen. Wenn die Geschwindigkeit eines Musters von Null auf einen konstanten Wert erhöht wird, zeigt der Reichardt-Detektor ein vorübergehendes Überschwingen bei der zeitlichen Frequenz der Musterbewegung, bevor er ein Gleichgewicht erreicht. Ein
Kontrast des bewegten Musters und vom Kontrast des Musters vor Bewegungsbeginn abhängt. Diese Befunde führten zu einem erweiterten Reichardt-Detektor, bei dem an den Querleitungen Hochpassfilter mit schnell adaptierenden Zeitkonstanten eingefügt wurden. Mit diesen Modifikationen kann das Reichardt-Modell sowohl den Gleichgewichtszustand, als auch sämtliche transienten Antworteigenschaften der Tangentialzellen in der Lobulaplatte detailliert erklären. Dies gilt nicht nur für Antworten auf schrittweise Geschwindigkeitsänderungen, sondern auch für Antworten auf Reize mit Gauss-verteilten Geschwindigkeitsprofilen (weißes Rauschen). Bei Verwendung solcher Reize mit verschiedenen Standardabweichungen zeigte die Antwort eine Anpassung der Geschwindigkeitskennlinie: die Steigung der Antwort-Geschwindigkeit-Funktion war umso steiler, je kleiner die Geschwindigkeitsschwankungen waren. Erstaunlich und völlig uneingängig ist die Tatsache, dass der Reichardt - Detektor die Geschwindigkeitskennlinie automatisch anpasst, auch wenn seine Filter keinerlei adaptiven Eigenschaften haben. Obwohl die Tangentialzellen die Ausgangssignale lokaler Bewegungsdetektoren räumlich integrieren und deshalb deren aufsummiertes Ausgangssignal repräsentieren sollten, können die Signale individueller Bewegungsdetektoren experimentell beobachtet werden, wenn eine räumliche Integration verhindert wird, indem man die Musterbewegung durch einen Schlitz präsentiert. Auch lokale Kalziummessungen an den feinen dendritischen Verästelungen erlauben die Messung individueller Bewegungsdetektoren. Mit beiden Methoden wurden lokale Signale nachgewiesen, die alle Eigenschaften von lokalen Bewegungsdetektoren gemäß dem Reichardt-Typ aufweisen (Egelhaaf et al. 1989).

All diese Befunde beweisen klar, dass die Tangentialzellen der Lobulaplatte von Fliegen Eingang von einer großen Anzahl lokaler Bewegungsdetektoren des ReichardtTyps erhalten. Die geringe Größe der Säulenelemente des optischen Lobus erschwert allerdings direkte elektrophysiologische Ableitungen und damit die Feststellung, welche der vielen Zellen an dem Schaltkreis beteiligt sind, der diesen Algorithmus implementiert. Dies hat sich in letzter Zeit vor allem durch das große Arsenal genetischer Hilfsmittel, die für Drosophila zur 
Verfügung stehen, geändert. Kurz gesagt, erlaubt das Gal4-UAS-System die gezielte Expression eines bestimmten Effektor-Gens in einer genetisch definierten Subpopulation von Neuronen. Das Effektor-Gen kann ein genetisch codierter Kalziumanzeiger für optische Ableitungen sein, ein K-Kanal mit hoher Leitfähigkeit zum Hyperpolarisieren der Neurone oder ein verändertes, dominant negatives Allel des Dynamin codierenden Gens shibire, um die Transmitterfreisetzung aus diesen Neuronen zu unterdrücken. Die Kombination dieser Techniken mit wholecell patch-Ableitungen an Tangentialzellen der Lobulaplatte erwies sich als eine sehr leistungsfähige Herangehensweise, die es ermöglichte, diejenigen Neurone zu identifizieren, die den Eingang zu den ReichardtDetektoren bzw. deren Ausgangssignal liefern.

In der Lamina sind die Photorezeptoren direkt oder indirekt mit fünf verschiedenen Lamina-Neuronen synaptisch verschaltet. Um zu entscheiden, welche dieser LaminaNeurone in den Schaltkreis für Bewegungsdetektion involviert sind, wurden an
Drosophila die Tangentialzellen abgeleitet, während die Ausschüttung chemischer Transmitter von L1- oder L2-Neuronen genetisch durch zellspezifische Expression von shibire blockiert wurde (Joesch et al. 2010). Die Blockade des Ausgangssignals von entweder L1- oder L2-Neuronen führte $\mathrm{zu}$ einer schwächeren, aber immer noch signifikanten Antwort der Tangentialzellen der Lobulaplatte auf ein bewegtes Muster. Die gleichzeitige Blockade der L1- und der L2-Zellen löschte jegliche Antwort der Tangentialzellen auf bewegte Reize vollständig aus. Diese Befunde wiesen in Übereinstimmung mit Verhaltensexperimenten darauf hin, dass die L1- und L2-Neurone die Haupteingangssignale für den Schaltkreis zur Bewegungswahrnehmung liefern (Rister et al. 2007). Eine klare funktionelle Spezialisierung war zunächst aber nicht offensichtlich. Als man aber statt Mustern Helligkeitsstufen mit entgegengesetzter Polarität bewegte, zeigte sich, dass die L1-Neurone $\mathrm{zu}$ einem ON-Schaltkreis projizieren, die L2-Neurone zu einem OFF-Schaltkreis. Eine L1-Blockade hob die Antworten auf bewegte ON-Kanten vollständig und selektiv auf, eine L2-Blockade die Antworten auf bewegte OFF-Kanten. In einer anderen Untersuchung, in der das Verhalten der Tiere statt der Antworten der Tangentialzellen gemessen wurde, wurde ein ausgeglichener Bewegungsreiz geboten, in dem sich eine ON-Kante in die eine Richtung bewegte und eine OFF-Kante in die entgegengesetzte Richtung. Fliegen mit blockiertem L1-Signal folgten der Bewegung der OFF-Kante, Fliegen mit blockiertem L2-Signal folgten der ON-Kante. Intakte Kontrollfliegen bewegten sich in der Regel gar nicht.

Diese Befunde zeigen, dass bei der Fliege die Signale der Photorezeptoren R1-6, die von den L1 bzw. den L2-Zellen übertragen werden, in der Lamina auf zwei getrennte $\mathrm{ON}$ - und OFF-Verarbeitungswege aufgeteilt werden (Abbildung 2C). Dies ist analog zu den Verhältnissen in der Vertebraten-Retina, wo Zapfen sowohl ON- als auch OFFBipolarzellen kontaktieren (siehe Euler und Hausselt, dieses Heft). In der VertebratenRetina erfolgt die Aufspaltung allerdings durch verschiedene Glutamatrezeptoren auf

\section{Hertie-Stiftung}

Die Gemeinnützige Hertie-Stiftung ist tätig in den Bereichen Vorschule, Schule, Hochschule und Neurowissenschaften. Sie ist der größte private Förderer neurowissenschaftlicher Forschung in Deutschland.

Zur Förderung der Forschung erfahrener Neurowissenschaftler und Neurowissenschaftlerinnen sowie als Auszeichnung für langjährige Spitzenleistung schreibt die Gemeinnützige Hertie-Stiftung die

\section{Hertie-Senior-Forschungsprofessur Neurowissenschaften $2013(\mathrm{~m} / \mathrm{w})$}

aus. Die Stiftungsprofessur ist für herausragende Neurowissenschaftler gedacht, welche die abschließenden Jahre ihrer beruflichen Laufbahn ausschließlich der Forschung widmen wollen und hat einen Förderumfang von 1 Mio. Euro. Sie hat eine Laufzeit von max. acht Jahren, ist bis zum Eintritt in den Ruhestand befristet und entsprechend den jetzigen Bezügen des Kandidaten dotiert (einschließlich der Leistungen für Pensionsansprüche, Krankenversicherung etc.). Weiterhin ist ein Zuschlag für außergewöhnliche Forschungsleistungen vorgesehen. Dem Inhaber der Stiftungsprofessur werden Arbeitsmöglichkeiten (Laborräume, Forschungsbudget etc.) nach dessen Vorstellungen und in Absprache mit der Universität bis zur Pensionierung sowie nach Antrag und Evaluation bis zu fünf Jahre danach zur Verfügung gestellt.

Bedingungen für Bewerber sind neben dem Nachweis der neurowissenschaftlichen Exzellenz ein Lebensalter von mindestens 59 Jahren und die Verpflichtung, mit Antritt der Stiftungsprofessur alle Leitungs- und Verwaltungsfunktionen an der von ihm bisher geleiteten Institution abzugeben, sich bis zur Pensionierung an der Lehre mit mindestens 3 Semesterwochenstunden zu beteiligen und neurowissenschaftliche Forschung zu betreiben. Bewerber sollten wünschenswerterweise noch mindestens drei Jahre bis zum geplanten Eintritt in den Ruhestand haben. Weitere Informationen sind unter www.ghst.de/seniorprofessur zu erhalten.

Der Kandidat für die Stiftungsprofessur wird zunächst in einem vertraulichen, personenbezogenen Verfahren durch eine Jury führender Neurowissenschaftler bestimmt. Danach werden in Absprache mit dem ausgewählten Kandidaten die Verhandlungen mit der Universität auf Realisierung der Stiftungsprofessur und ihre Ausstattung von der Hertie-Stiftung geführt. Die endgültige Entscheidung über die Vergabe der Professur trifft der Vorstand der Gemeinnützigen Hertie-Stiftung. Die Verleihung erfolgt 2013 in Frankfurt.

Bewerbungen und Rückfragen sind bis zum 1. November 2012 an folgende Adresse zu richten:

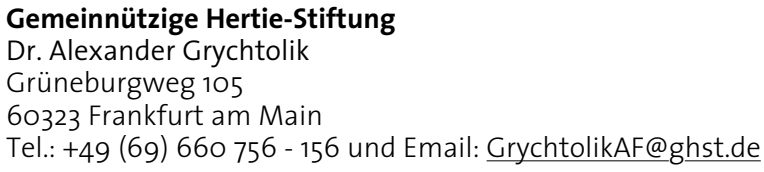


den ON- und den OFF-Bipolarzellen, sodass ON-Bipolarzellen durch Licht depolarisiert, OFF-Bipolarzellen aber hyperpolarisiert werden. Bei Fliegen ist die Antwort in den Dendriten von L1- und L2-Neuronen identisch und besteht aus einer transienten $\mathrm{Hy}$ -

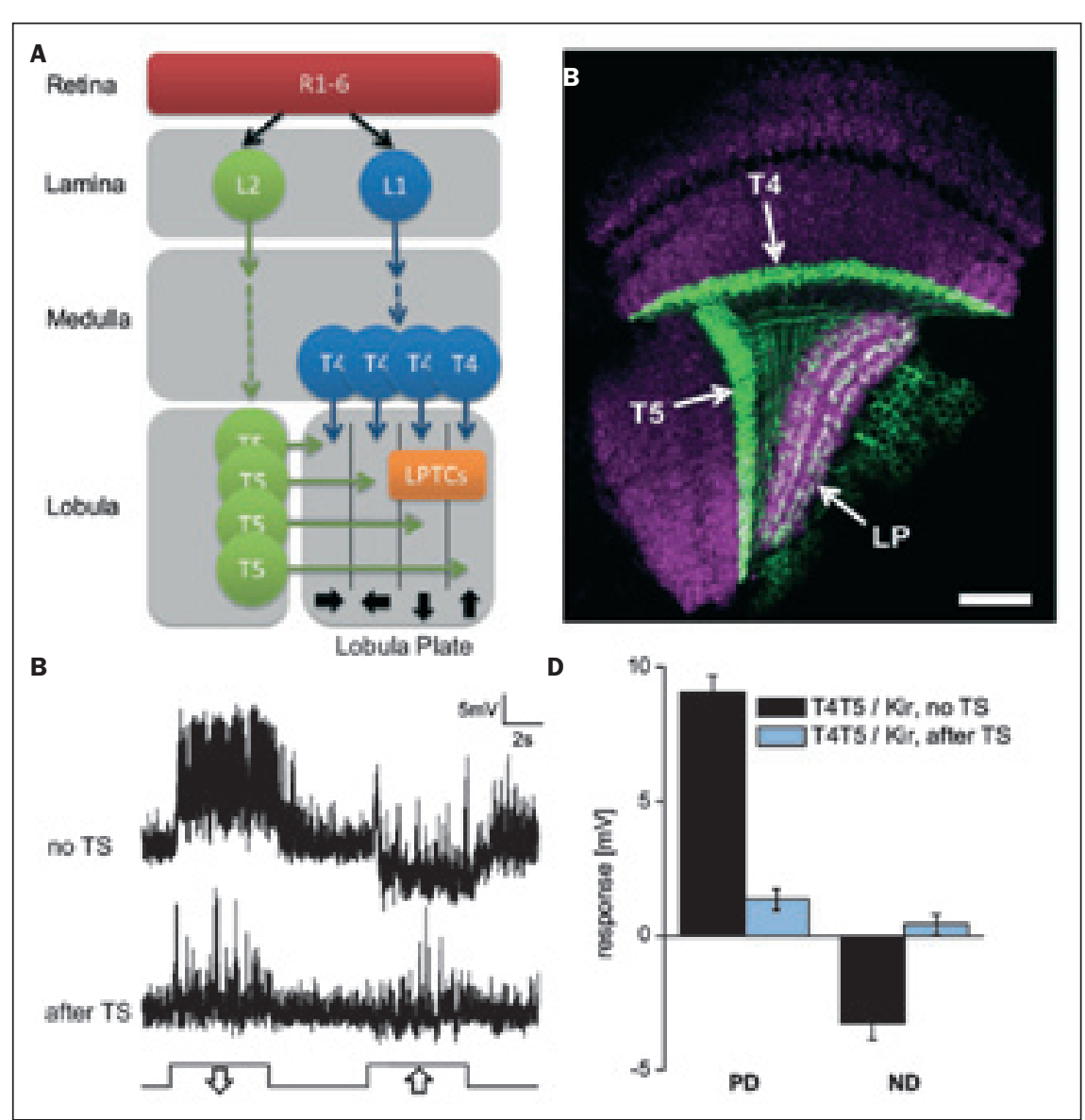

Abb. 3: T4- und T5- Zellen stellen die Ausgangsstrukturen der lokalen Bewegungsdetektoren dar (aus Schnell et al. 2012).

A): Schematisierte Verarbeitungswege, die von den Photorezeptoren R1-6 zu den Tangentialzellen der Lobulaplatte führen. In der Lamina wird das Signal der Photorezeptoren R1-6 in zwei parallele Verarbeitungswege L1 und L2 aufgeteilt. Ihre Signale werden von einigen bisher nicht identifizierten Neuronen verarbeitet und auf T4- und T5- Zellen geleitet. Es gibt je vier T4- und T5- Typen, die sich jeweils in einer von vier Schichten der Lobulaplatte verzweigen und die Dendriten der Tangentialzellen kontaktieren. LPTC: Tangentialzellen der Lobulaplatte. B): Einzelner horizontaler optischer Schnitt durch den optischen Lobus einer Fliege, die unter der Kontrolle der Gal4 -Treiberlinie R42F06 in den T4- und den T5-Zellen Kir2.1-EGFP exprimiert. LP: Lobulaplatte, Maßstabsbalken entspricht $20 \mu \mathrm{m}$.

C): Antwort einer frontalen Zelle des vertikalen Systems auf eine Abwärts- (Vorzugsrichtung PD) und eine Aufwärtsbewegung (Nullrichtung ND) eines Sinusgitters (Zeitfrequenz = $1 \mathrm{~Hz}$ ) bei einer Kontrollfliege (keine Temperaturveränderung „TS“, oben) und bei einer experimentellen Fliege (mit Temperaturveränderung, unten). Beide Fliegen hatten denselben Genotyp. Bei den experimentellen Fliegen wurde die Expression von Kir in T4- und T5-Zellen durch eine Temperaturveränderung ausgelöst. Bei der experimentellen Fliege ist die Antwort auf den bewegten Reiz fast völlig aufgehoben. D): Mittlere Antworten auf Bewegung in Vorzugs- (PD) und Nullrichtung (ND) (wie in C gezeigt) von Kontrollfliegen ohne Temperaturveränderung (schwarze Balken, $n=4$ ) und experimentellen Fliegen (blaue Balken, $n=7$ ) nach einer Temperaturveränderung. Die Antworten auf bewegte Reize sind hier stark reduziert. Die Fehlerbalken zeigen die Standardfehler an.
Licht entweder am Axonterminal entsteht, wie es an L2-Neuronen durch Ca-Messungen nachgewiesen wurde, oder erst in nachgeschalteten Neuronen.

Aus dem Befund, dass der ON- und der OFF-Verarbeitungsweg getrennte Eingänge erhalten, ergibt sich die interessante Frage, wie viele Typen von Bewegungsdetektoren das Fliegengehirn eigentlich hat. Gibt es vier verschiedene Detektoren, einen für jede Reizkombination (ON-ON, OFF-OFF, ON-OFF, OFF-ON) oder nur zwei (ONON, OFF-OFF)? Diese Frage kann man direkt mit einem Stimulus untersuchen, bei dem die Helligkeit zweier benachbarter Balken schrittweise und nacheinander von einem mittleren Niveau, das auch in der Umgebung vorhanden ist, entweder erhöht (ON) oder abgeschwächt (OFF) wird. Präsentiert man den Fliegen solche Reize, so findet man sowohl bei Ableitungen von Tangentialzellen der Lobulaplatte als auch bei Verhaltensmessungen positive Antworten auf ON-ON und OFF-OFF Sequenzen und negative Antworten auf ON-OFF und OFF-ON Sequenzen (Eichner et al. 2011). Obwohl diese Ergebnisse scheinbar klar auf vier Detektorsubtypen hinweisen, zeigte eine sorgfältige quantitative Modellierung, die auch periphere Filterstadien in Betracht zog, dass Antworten auf gemischte Helligkeitsstufen auch von nur zwei Detektoren (ON-ON und OFF-OFF) geleistet werden können, sofern eine Restinformation über die mittlere Helligkeit im Eingangssignal des Bewegungsdetektors erhalten bleibt. Eine selektivere Reizsequenz aus kurzen Helligkeitspulsen statt aus Helligkeitsstufen ergab ausschließlich Antworten auf Sequenzen mit gleichem Vorzeichen (ON-ON und OFF-OFF), was die Existenz von Bewegungsdetektoren mit gemischtem Vorzeichen ausschließt (Eichner et al. 2011).

Mit diesen Befunden konnten die Neurone identifiziert werden, die den Eingang zu den Schaltkreisen für Bewegungsdetektion liefern. Sie zeigten außerdem, dass die einfache Multiplikation, die im Algorithmus definiert ist, neuronal durch zwei getrennte Nichtlinearitäten implementiert ist. Eine der beiden ist ausschließlich mit positiven, die andere mit negativen Eingangssignalen befasst. Dadurch entstehen zwei parallele Verarbeitungswege für Bewegungsdetektion (Figur 2C). Aber wie sieht es mit dem Ausgang des Bewegungsdetektors aus? Welche Neurone im optischen Lobus der Fliege leiten diese Signale an die Tangentialzellen der Lobulaplatte weiter?

Es wurde zunächst gezeigt, dass Tangentialzellen von Drosophila exzitatorischen und inhibitorischen Eingang von lokalen 
bewegungsempfindlichen Elementen mit entgegengesetzter Vorzugsrichtung erhalten. Dafür wurde depolarisierender und hyperpolarisierender Strom während einer Reizbewegung in Vorzugs- und Nullrichtung in die Tangentialzelle injiziert. Wenn depolarisierender Strom injiziert wird, wird die Antwort auf Bewegung in Vorzugsrichtung kleiner und die Antwort auf Bewegung in Nullrichtung größer. Während einer Injektion von hyperpolarisierendem Strom wird die Antwort auf Bewegung in Vorzugsrichtung größer, diejenige auf Bewegung in Nullrichtung kleiner. Dies kann man nur mit der Annahme erklären, dass die Zelle zwei synaptische Eingänge erhält, deren Umkehrpotenziale oberhalb und unterhalb des Ruhepotenzials der Zelle liegen. Deshalb führt Injektion von depolarisierenden und hyperpolarisierenden Strömen zu einer Reduktion der Triebkraft des einen und einer Verstärkung der Triebkraft des anderen Eingangs. Diese Ergebnisse deuten darauf hin, dass die Subtraktionsebene im Reichardt - Detektor innerhalb der Dendriten der Tangentialzellen liegt. Ältere Experimente an Tangentialzellen von Schmeißfliegen kamen zu ähnlichen Ergebnissen.

Aber von welchen Neuronen stammen diese exzitatorischen und inhibitorischen Eingänge zu den Tangentialzellen der Lobulaplatte? Aus vielerlei Gründen wurden die durch buschige Dendriten gekennzeichneten T-Zellen (bushy T-cells) seit Langem verdächtigt, die Eingangsstrukturen für die Tangentialzellen der Lobulaplatte zu sein (Fischbach und Dittrich 1989). Es gibt vier verschiedene Typen von T4-Zellen pro Säule, die sich in der am weitesten proximal gelegenen Schicht der Medulla verzweigen und diese mit jeweils einer von vier Unterschichten der Lobulaplatte verbinden (Abbildung 3A). In ähnlicher Weise verbinden vier Typen von T5-Zellen die am weitesten posterior gelegene Schicht der Lobula mit jeweils einer der vier Unterschichten der Lobulaplatte. Mit der 2-Deoxyglucose (2DG) - Methode konnte Buchner et al. (1984) nach Reizung mit bewegten Mustern in Abhängigkeit von der Richtung des bewegten Reizes in einer der vier Schichten der Lobulaplatte eine starke Markierung nachweisen. Die Bewegungsrichtung, die eine bestimmte Schicht aktiviert und deshalb zu einer 2-DG - Markierung führt, stimmt mit der Vorzugsrichtung der Tangentialzellen überein, deren Dendriten sich in dieser Schicht verzweigen. Neben der Lobulaplatte war die am weitesten proximal gelegene Schicht der Medulla, wo sich die T4-Zellen verzweigen, sowie die am weitesten posterior gelegene Schicht der Lamina, wo sich die T5-Zellen verzweigen, am stärksten 2-DG markiert (Buchner et al. 1984). Schließlich zeigte eine elektronenmikroskopische Studie an der Schmeißfliege eindeutig eine chemische Synapse zwischen einem Dendriten einer Horizontal-System (HS)-Zelle und einer T4-Zelle. Aufgrund ihrer geringen Größe konnten die visuellen Antworteigenschaften der T4- und T5-Zellen nur in Einzelfällen untersucht werden: Die wenigen erfolgreichen Ableitungen zeigten, dass T5-Zellen richtungsselektiv, T4-Zellen aber richtungsunspezifisch sind. Letztendlich haben aber all diese Befunde nicht bewiesen, dass die T4- und T5-Zellen tatsächlich das richtungsselektive Eingangssignal für die Tangentialzellen liefern.

Dieser Beweis wurde kürzlich in einer Studie an Drosophila geführt, in der eine für T4- und T5-Zellen selektive Treiberlinie benutzt wurde (Abbildung 3B). Wie bei der Identifizierung der L1- und L2-Neurone als Eingangsstrukturen des Schaltkreises für Bewegungserkennung wurde diese Linie hier benutzt, um T4- und T5-Zellen selektiv zu blockieren, während gleichzeitig die Antwort der Tangentialzellen der Lobulaplatte auf bewegte Reize mit einem whole cell Patch am Soma abgeleitet wurde. In dem in Abbildung 3C, D gezeigten Beispiel wurde ein einwärts gleichrichtender K-Kanal („KIR“) in T4- und T5-Zellen exprimiert, um die betroffenen Neurone an ihrem Ruhepotenzial zu klemmen. Damit kombiniert wurde die Expression von Gal80, einem temperaturempfindlichen Inhibitor von Gal4. Bei Raumtemperatur unterdrückt Gal80 die Wirkung von Gal4. Daher werden keine KIR-Kanäle exprimiert. Unter diesen Bedingungen beobachtet man eine normale Depolarisation bei Bewegung in der Vorzugsrichtung und eine Hyperpolarisation bei Bewegung in Nullrichtung (Abbildung 3C oben, ,no TS“ keine Temperaturveränderung). Wenn die Fliegen allerdings zwei Tage vor dem Experiment einer Temperaturveränderung ausgesetzt werden, kann keine richtungsselektive Antwort abgeleitet werden (Abbildung 3C unten, ,TS“ Temperaturveränderung). Diese Befunde (Mittelwerte in Abbildung 3D) zeigen eindeutig, dass die T4- und T5-Zellen den richtungsselektiven Eingang zu den Tangentialzellen der Lobulaplatte darstellen (Schnell et al. 2012).

Aufgrund dieser Ergebnisse und den Hinweisen auf Verarbeitungswege, die L1mit T4- und L2- mit T5-Zellen verbinden, geht man derzeit davon aus, dass es bei der Fliege zwei parallel arbeitende Systeme für Bewegungswahrnehmung gibt. Ein System analysiert die Bewegung von Helligkeitszunahmen, das andere die Bewegung von Helligkeitsabnahmen. Beide Verarbeitungs- wege vereinigen sich an den Dendriten der Tangentialzellen der Lobulaplatte und versehen sie mit Information über die lokale Bewegungsrichtung an jeder Position im Sehfeld.

\section{Globale Analyse des optischen Flusses in der Lobulaplatte}

Die lokale Bewegungsdetektion ist der erste Schritt in der Analyse des optischen Flusses. Sie liefert dem Nervensystem ein Vektorfeld, das durch die Ausgangssignale vieler retinotop angeordneter Bewegungsdetektoren des Reichardt - Typs repräsentiert wird. Diese Information über den optischen Fluss wird dann von Tangentialzellen innerhalb der Lobulaplatte weiterverarbeitet. All diese Zellen haben große Dendriten, mit denen sie die Signale verschiedener Subpopulationen von Bewegungsdetektoren räumlich integrieren. Entsprechend ihrer Vorzugsrichtung werden sie in horizontale (H-) und vertikale (V-) Zellen eingeteilt. Die Dendriten der Zellen des horizontalen Systems verzweigen sich in der anterioren Schicht der Lobulaplatte. Die am besten untersuchten Vertreter dieser Gruppe sind die drei HS-Zellen (Schnell et al. 2010). Das vertikale System besteht aus zehn VS -bei großen Fliegenarten und mindestens sechs VS-Zellen bei Drosophila. Die Dendriten der VS-Zellen sind in der posterioren Schicht der Lobulaplatte entlang der dorso-ventralen Achse ausgerichtet. Die VS-Zellen werden entsprechend der Lage ihrer Dendriten von lateral nach proximal durchnummeriert.

Aufgrund der retinotopen Organisation kann man anhand der Lage der Dendriten einer Zelle innerhalb der Lobulaplatte gut vorhersagen, wo das Zentrum ihres rezeptiven Feldes liegt. Die rezeptiven Felder der HSZellen, die den nördlichen Teil (HSN), den Äquator (HSE) und den südlichen Teil (HSS) der Lobulaplatte bedecken, liegen im dorsalen, mittleren und ventralen Gesichtsfeld der Fliege. Sogar innerhalb eines Dendriten einer Einzelzelle tritt die retinotope Organisation der Lobulaplatte zu Tage, wenn man lokale Bewegungsreize an verschiedenen Stellen innerhalb des rezeptiven Feldes präsentiert und die Aktivität des Dendriten mit Ca-Imaging darstellt. Allerdings entdeckten Krapp und seine Kollegen (Krapp et al. 1998) bei der detaillierten Untersuchung der Tangentialzellen der Schmeißfliege, dass sich deren rezeptive Felder entlang des Azimut viel weiter ausdehnen, als es aufgrund ihres dendritischen Feldes in der Lobulaplatte zu erwarten gewesen wäre. Außerdem fanden sie, dass das rezeptive Feld aus Zonen mit verschiedener Vorzugsrichtung zusam- 


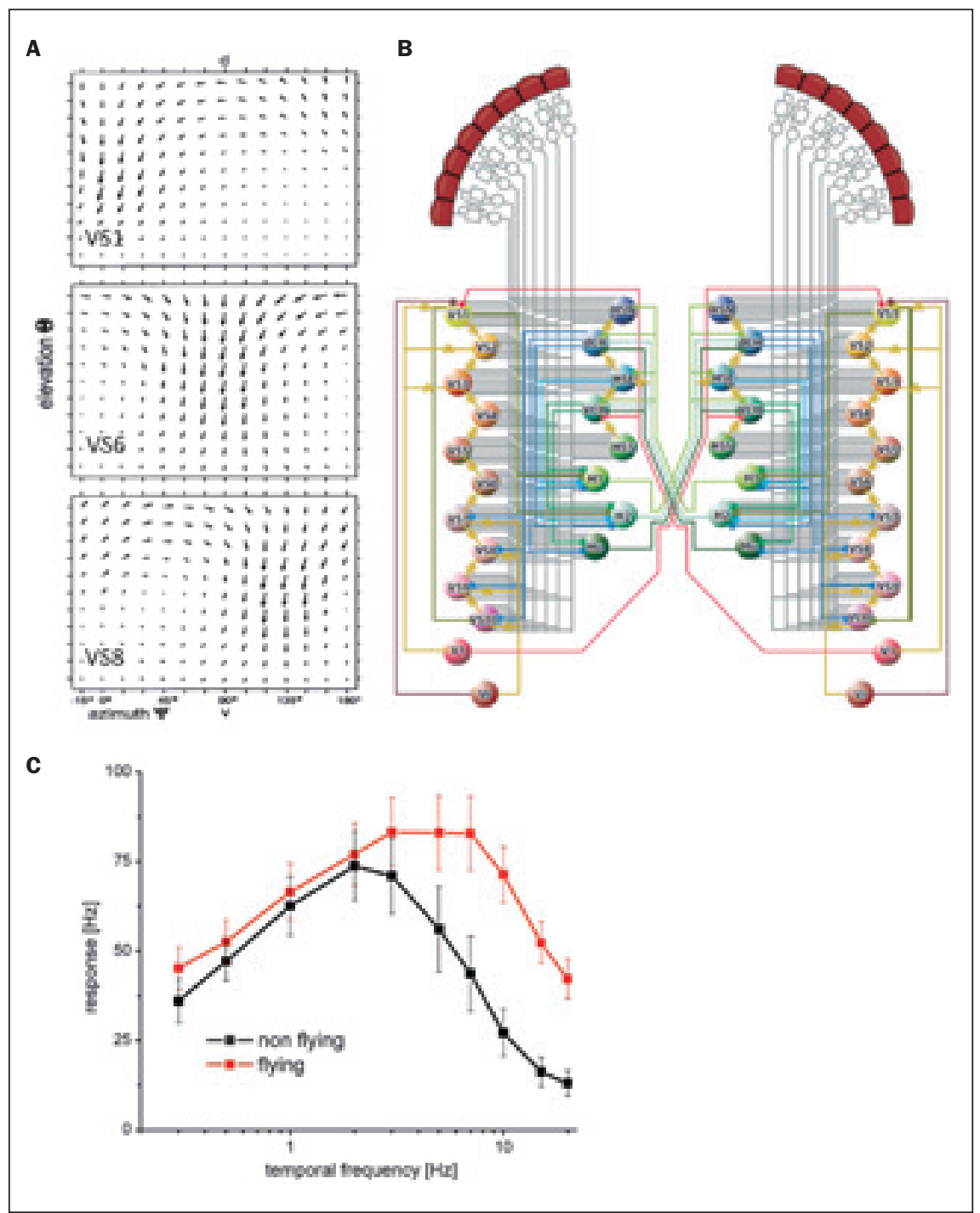

Abb. 4 A): Rezeptive Felder der drei Zellen des vertikalen Systems (VS-Zellen) der Schmeißfliege Calliphora vicina (aus Krapp et al. 1998).

B): Verschaltungsschema der Tangentialzellen der Lobulaplatte der Schmeißfliege. Exzitatorische Synapsen sind durch Dreiecke, inhibitorische Synapsen durch Kreise, und elektrische Synapsen durch ein Widerstandssymbol gekennzeichnet. Beachte, dass die VS-Zellen einer jeden Hemisphäre untereinander sequenziell über elektrische Synapsen verbunden sind. C): Vergleich der Geschwindigkeitstuningkurven einer H1 -Tangentialzelle bei fliegenden (rot) und stationäen (schwarz) Fliegen. Ordinate: Antwortstärke (response) in Hz, Abszisse: zeitliche Frequenz (temporal frequency, d.h. Geschwindigkeit dividiert durch die räumliche Wellenlänge des Reizmusters) in Hz. Bei fliegenden Tieren erweitert sich die Tuningkurve zu höheren Geschwindigkeiten hin.

mengesetzt ist. Diese Eigenschaft ist in Abbildung 4A durch die Lage und Richtung der Pfeile für drei verschiedene VS-Zellen dargestellt. Die rezeptiven Felder der VSZellen zeigen entsprechend ihrer Lage in der Lobulaplatte eine maximale Empfindlichkeit für Bewegung nach unten. Zusätzlich reagieren sie auf horizontale Bewegung im dorsalen Teil des Gesichtsfeldes und auf Bewegung nach oben in einem Bereich, der entlang des Azimut ca. $180^{\circ}$ vom Bereich der höchsten Empfindlichkeit entfernt ist. Insgesamt sehen die rezeptiven Felder wie Vektorfelder aus, die dann auftreten, wenn sich das Tier um eine bestimmte Körperachse dreht. Da alle Zellen unterschiedliche rezeptive Felder haben, entstand die Vorstellung, dass die Tangentialzellen abgestimmte Filter darstellen, von denen jeweils eine bei einem bestimmten Manöver der Fliege maximal reagiert. Dies konnte tatsächlich experimentell nachgewiesen werden.
Diese Beobachtung stellt die Tangentialzellen der Lobulaplatte in das Zentrum der visuellen Kurskontrolle. Es bleibt aber die Frage, wie diese rezeptiven Felder entstehen. Wenn diese Zellen isoliert und streng parallel arbeiten würden, müssten ihre rezeptiven Felder viel kleiner sein. Außerdem würde man erwarten, dass ihr Eingang von elementaren Bewegungsdetektoren eine mehr oder weniger einheitliche Vorzugsrichtung hat, da sich die meisten Zellen nur in einer einzigen Schicht der Lobulaplatte verzweigen. Dieses Problem wurde durch eine Reihe von Experimenten gelöst, in denen zwei Tangentialzellen simultan abgeleitet wurden (Haag und Borst 2004). In diesen Experimenten wurde in eine Zelle Strom injiziert und die Reaktion auf diese Injektion in der jeweils anderen Zelle registriert. Diese und andere Experimente haben ein faszinierendes Netzwerk in der Lobulaplatte zu Tage gefördert, in dem die meisten Tangentialzellen miteinander verbunden sind, sowohl innerhalb einer Hemisphäre, als auch zwischen beiden Hemisphären. Viele dieser Verbindungen beruhen auf elektrischen, statt auf chemischen Synapsen. Diese Verbindungen werden für die großen und komplexen rezeptiven Felder verantwortlich gemacht: Während ein Teil des rezeptiven Feldes direkt über die Dendriten entsteht, wird zusätzliche Information indirekt über die Nachbarzellen vermittelt. Wenn man also bestimmte Zellen der Lobulaplatte eliminiert, sollten sich die rezeptiven Felder der verbliebenen Zellen verändern. Wie durch detaillierte Computersimulationen korrekt vorhergesagt, veränderten sich tatsächlich die rezeptiven Felder der restlichen Zellen, wenn bei der Schmeißfliege eine einzelne Tangentialzelle experimentell mittels Photoablation eliminiert wurde. Die Computersimulationen sagten auch voraus, dass aufgrund der elektrischen Kompartimentierung der VS-Zellen und der spezifischen Kontakte benachbarter VS-Zellen am Axonterminal unterschiedliche rezeptive Felder im Dendriten und am Axonterminal auftreten sollten. Diese Vorhersage konnte tatsächlich in Ca-Imaging Studien bestätigt werden. In jüngster Zeit wurden in einer groß angelegten Simulation unter Berücksichtigung aller verfügbarer Daten über die Verbindungen zwischen 22 Tangentialzellen in der Lobulaplatte in jeder Hemisphäre der Schmeißfliege rezeptive Felder konstruiert, die den natürlichen rezeptiven Feldern der Tangentialzellen sehr nahe kommen. Zusammengefasst gibt es also eine Menge experimenteller Hinweise darauf, dass die rezeptiven Felder der Tangentialzellen der Lobulaplatte durch die dendritische Integration lokaler, bewegungssensitiver 
Eingangselemente, sowie durch die intraund interhemisphärischen Verbindungen der Tangentialzellen untereinander zustande kommen.

Offensichtlich ist ein großer Teil der Analyse des optischen Flusses auf der Ebene der Lobulaplatte bereits abgeschlossen. Im nächsten Schritt in Richtung Flugkontrolle treten die Tangentialzellen der Lobulaplatte in synaptischen Kontakt mit absteigenden Neuronen, die entweder mit den motorischen Zentren im Thorakal-Ganglion in Verbindung stehen, oder direkt Halsmuskeln innervieren und somit Kopfbewegungen steuern. Zwei solcher Neurone, DNOVS1 und DNOVS2 (Descending Neurons of the Ocellar and Vertical System) wurden in letzter Zeit detailliert untersucht. Durch Strominjektionen während Doppelableitungen von DNOVS-Zellen und verschiedenen VS-Zellen wurden Verbindungen zwischen diesen Zelltypen nachgewiesen. Anscheinend reagieren die beiden DNOVS-Zellen, ähnlich wie die VS-Zellen, von denen sie ihren Eingang erhalten, auf zwei verschiedene Rotationsachsen besonders empfindlich. Auch die Tuningbreite der DNOVS-Zellen ähnelt derjenigen von VS-Zellen, die mit ihnen in Verbindung stehen. Während der Rotation von natürlichen Bildern reagieren DNOVS-Zellen gleichmäßig, die Signale der VS-Zellen fluktuieren dagegen erheblich über die Zeit. Dieser Effekt rührt von den axo-axonalen elektrischen Synapsen zwischen den VS-Zellterminalen her, die die Ausgangssignale linear interpolieren. Dies ist am Membranpotenzial der postsynaptischen Zellen deutlich zu erkennen. Es nimmt daher von der Lobulaplatte zu den absteigenden Neuronen nicht die Selektivität für bestimmte optische Flussfelder zu, sondern die Robustheit der Antworten bei verschiedenen Gegebenheiten der visuellen Umgebung. Da jedoch bisher nur wenige absteigende Neurone genau untersucht wurden, sollten diese Befunde zum jetzigen Zeitpunkt nicht verallgemeinert werden.

All die hier beschriebenen Eigenschaften der Tangentialzellen machen sie zu idealen Kandidaten für die neuronale Steuerung der optomotorischen Reaktion. Werden Tangentialzellen operativ oder durch Genmutationen ausgeschaltet, ist die entsprechende optomotorische Reaktion stark beeinträchtigt. Allerdings werden bei solchen Läsionsstudien immer eine große und schlecht definierte Gruppe von Tangentialzellen ausgeschaltet. Die Auswirkungen der Ausschaltung einzelner Tangentialzellen auf die Drehreaktion von fixierten Tieren wurden nie gemessen. Ein Befund, der die Rolle der Tangentialzellen bei der Kontrolle der optomotorischen Antwort ernsthaft in Frage stellte, war die Tatsache, dass die optomotorische Antwort von Schmeiß- und Taufliegen bei wesentlich höheren zeitlichen Frequenzen ein Optimum zeigt als die Tangentialzellen. Da bei einer räumlichen Mittelung die Information über die zeitliche Frequenz des Reizes verloren geht, kann dieser Unterschied auch nicht durch eine Art Nachverarbeitung erzielt werden. Neuere Untersuchungen haben nun gezeigt, dass die Tangentialzellen bei am Thorax befestigten laufenden oder fliegenden Tieren ihre Antworteigenschaften stark ändern. Sie erhöhen nicht nur ihre Spontanaktivität und allgemeine Antwortamplitude, auch die optimale zeitliche Frequenz verschiebt sich hin zu höheren Frequenzen (Abbildung 4C). Mechanistisch kann dies durch die Freisetzung von Oktopamin erklärt werden, wodurch die Zeitkonstante des Verzögerungsfilters des elementaren Bewegungsdetektors verkürzt wird. Diese Befunde eliminieren eine Diskrepanz zwischen den Antworteigenschaften der Tangentialzellen und der optomotorischen Reaktion. Dennoch sollte man nicht vergessen, dass die Hinweise auf die Tangentialzellen als Kontrollinstanz der optomotorischen Reaktion bisher nur auf Korrelationen beruhen.

\section{Schlussfolgerungen}

Die visuelle Kurskontrolle bei Fliegen wird schon seit fast einem halben Jahrhundert untersucht, aber trotz erheblicher Fortschritte scheint kein Ende in Sicht zu sein. Das aktuelle Interesse an dieser Fragestellung konzentriert sich auf Drosophila, sowohl was die Identifizierung der Verarbeitungswege für Bewegungsdetektion angeht, als auch bezüglich der Steuerung verschiedener Verhaltenselemente. Die Kombination aus hochentwickelter Genetik mit Elektrophysiologie, Ca-Imaging und technisch ausgefeilten, quantitativen Verhaltensmessungen wird unser Verständnis der neuronalen Grundlagen der visuellen Kurskontrolle bei der Fliege enorm vorantreiben.

\section{Literatur}

Buchner, E., Buchner, S. und Bülthoff, I. (1984): Deoxyglucose mapping of nervous activity induced in Drosophila brain by visual movement. J Comp Physiol A 155: 471-483.

Egelhaaf, M., Borst, A. und Reichardt, W. (1989): Computational structure of a biological motion detection system as revealed by local detector analysis in the fly's nervous system. $J$ Opt Soc Am A 6: 1070-1087.

Eichner, H., Joesch, M., Schnell, B., Reiff, D.F. und Borst, A. (2011): Internal structure of the fly elementary motion detector. Neuron 70 : 1155-1164.

Fischbach, K.F. und Dittrich, A.P.M. (1989): The optic lobe of Drosophila melanogaster. I. A Golgi analysis of wild-type structure. Cell Tissue Res 258: 441-475.

Haag, J. und Borst, A. (2004): Neural mechanism underlying complex receptive field properties of motion-sensitive interneurons. Nature Neurosci 7: 628-634.

Hassenstein, B. und Reichardt, W. (1956): Systemtheoretische Analyse der Zeit-, Reihenfolgenund Vorzeichenauswertung bei der Bewegungsperzeption des Rüsselkäfers Chlorophanus. $Z$ Naturforsch 11b: 513-524.

Joesch, M., Schnell, B., Raghu, S.V., Reiff, D.F. und Borst, A. (2010): ON and OFF pathways in Drosophila motion vision. Nature 468: 300-304.

Krapp, H.G., Hengstenberg, B. und Hengstenberg, R. (1998): Dendritic structure and receptivefield organization of optic flow processing interneurons in the fly. $J$ Neurophysiol 79: 1902-1917.

Rister, J., Pauls, D., Schnell, B., Ting, C.Y., Lee, C.H., Sinakevitch, I., Morante, J., Strausfeld, N.J., Ito, K. und Heisenberg, M. (2007): Dissection of the peripheral motion channel in the visual system of Drosophila melanogaster. Neuron 56: 155-170.

Schnell, B., Raghu, S.V., Nern, A. und Borst, A. (2012): Columnar cells necessary for motion responses of wide-field visual interneurons in Drosophila. J Comp Physiol A 198: 389-395.

Eine vollständige Literaturliste kann beim Autor angefordert werden.

\section{Kurzbiografie}

Prof. Dr. Alexander Borst: Geboren am 18. August 1957 in Bad Neustadt/S, Deutschland. Studium der Biologie, Universität Würzburg, Deutschland (19761981). Doktorarbeit bei Martin Heisenberg, Universität Würzburg, Deutschland (1984). Forschungsassistent, Max-Planck-Institut für Biologische Kybernetik, Tübingen (1984-1993). Nachwuchsgruppenleiter, Friedrich-Miescher-Labor der MPG, Tübingen (1993-1999). Professor, UC Berkeley (1999-2001). Direktor, Max-Planck-Institut für Neurobiologie, Martinsried (seit 2001).

\section{Korrespondenzadresse}

\section{Prof. Dr. Alexander Borst}

MPI für Neurobiologie

Systems and Computational Neurobiology

Am Klopferspitz 18

82152 Martinsried

Tel.: $\quad+498985782150$

Fax: $\quad+498985782152$

E-Mail: borst@neuro.mpg.de

(C) Springer-Verlag GmbH 2012 